\title{
Discussing Van Wyk's Theory[1] Concerning Compression of a Fibrous Mass
}

\author{
By Kazuo Wakayama* and Tatsukichi Hattori**, * ${ }^{*}$ EMBER, TMSJ \\ *, ** Faculty of Textile Fibers, Kyoto University of Industrial Arts and Textile Fibers, Kyoto
}

\begin{abstract}
Van Wyk's theory on the compression of a fibrous mass is refuted by the authors on the ground that it ignores the substantial thickness of a fiber. The authors' formula, which shows the theoretical relation between the pressure and volume of a compressed specimen, explains the experimental tendency of the pressure-volume curve. However, it offers no strict experimental verification, because there are unavoidable limitations on the method of experiment at present usually employed and the authors have used that method to verify their formula.

The following expression, which the authors believe useful for the theoretical treatment of the compression of a fibrous mass, has been obtained:

$$
\frac{\Delta l}{\Delta t-\Delta l}=\frac{1}{\lambda}\left(\omega_{0}+\text { Pressure }\right)
$$

where $\Delta t$ and $\Delta l$ are the apparent height and the substantial thickness of the elemental thin layer in a compressed specimen, and $\omega_{0}$ is the latent pressure of the specimen. This expression shows the proportional relation between the degree of packing, $\Delta l /(\Delta t-\Delta t)$, and the pressure in an elemental thin layer of a compressed specimen. The symbol $\lambda$ is named "the modulus of the degree of packing."
\end{abstract}

\section{Introduction}

Compressive property is one of the important elements in the appraisal of fibers and finished products. There are many published discussions on the subject, but almost all of them are experimental, not theoretical. A theoretical study of compressive property must begin with inquiry into the shape of the pressure and the volume curve of a compressed fibrous mass. Efforts have been made by many experts to derive a general equation for a mass of randomly oriented fibers-from the characters of the fibers constituting the mass. A typical example of such efforts is Van Wyk's theory. P. G. de Macarty has commented on it as "sophisticated," [2] but the authors do not belive the Van Wyk theory should be ignored in any thorough research into compressive property.

This article seeks to revise some points in Van Wyk's theory on which he sounds unconvincing. He takes up the thickness of a fiber for consideration only in estimating the mean length of the fiber and ignores the thickness in his inquiry into the compressive process. That is to say, he seems to think of a fibrous mass as an assemblage of segmental lines having a mean effective length.

Van Wyk's theory is not directly applicable to the usual test, because it assumes an equal pressure on layers of different heights of a compressed specimen. In actual tests, however, specimens encounter, just under the compressional piston, various obstacles to an equal pressure-such as friction of fibers with the contrainer wall and the lateral bulg- ing of the specimens.

The present article suggests what the authors believe a formula applicable to an elemental thin layer of any height in a compressed specimen. It shows how the constants in the formula can be expressed by the attributes of the fibers constituting the specimen.

\section{Synopsis of Van Wyk's theory}

Any revision of Van Wky's theory must be prefaced with a synopsis of it. He simplifies the definition of the compression of a fiber mass by saying that it consists only of the bending of fibers, and ignores their possible twisting, slippage and extension. An increase in bending deflection $\Delta y$ on a model beam having the span $2 b$ is connected with an increase in concentrated load $\Delta f$ by the relation

$$
\Delta f \propto \frac{I Y}{b^{3}} \Delta y
$$

where $Y$ is Young's modulus of elasticity and $I$ the moment of inertia for the circular cross section of a beam having the diameter $d$. To apply this model to fibers, a mass of fibers is regarded as a system consisting of the above-mentioned bending units $b$, which are the elements between adjacent contacts of fibers and which are always oriented at random during the process of compression.

The mean length of the elements is calculated as follows: 


$$
b=\frac{v}{n}=\frac{2 v}{\pi d L}
$$

where $L$ is the total length of the fibers of a compressed specimen in a container having a unit area of the cross section. Originally the reciprocal of $b$, multiplied by the height $v$ of the specimen, was derived by Van Wyk as the mean of frequency $n$ with which a sphere of the diameter $d$, which is equal to that of the fiber, encountered fibers when moved vertically in the container. According to Van Wyk's calculation, the value of $b$ is small enough for a statistical computation to be applicable to a fibrous mass.

As for the downward penetration of compressive pressure into a specimen, Van $W_{y k}$ sees fit to take notice of thin layers of thickness $c$ at an arbitrary height in the container. He thinks each elemental fiber in such layers takes a share of the compressive pressure and that the sum of those shares of the elements in a layer (and an increase $\Delta p$ in that sum) penetrates one layer after another to the bottom of the container. The aforesaid sum is equivalent to the compressive pressure on a fibrous mass.

In the Van Wyk theory, the thickness $c$ of the layers is the vertical height occupied by an elemental fiber. The number of elements in a layer is, therefore, shown by $(c / v) \cdot(L / b)$ and, accordingly, $\Delta p$ is given by $(c L / v b) \Delta f$. Here Van Wyk takes $\Delta f$ in equation (1) as aproximately equvalent to the shares of pressure by the elemental fibers. Then according to him, $\Delta p$ reduces each layer in thickness by an amount equal to (proportional to, to be more exact) $\Delta y$ in equation (1), so that the change $\Delta v$ in the height of the specimen is $\Delta v=-(v / c) \Delta y$.

Thus is established the equation:

$$
\Delta p \propto \frac{L}{b} \frac{c}{v} \cdot \Delta f \propto-\frac{I Y}{b^{3}} \frac{L}{b} \frac{c^{2}}{v^{2}} \cdot \Delta v \quad \cdots \cdots \cdots
$$

He obtains $b^{3} / 3$ as the mean value of $c$ with elements oriented at random. Substituting this value, equation (2) and the relation

$$
I=\frac{\pi p^{4}}{64} \quad \text { and } \quad \frac{\pi d^{2}}{4} L==\frac{m}{\rho}
$$

into equation (3), he derives:

$$
\Delta p \propto-\frac{I Y}{b^{3}} \frac{L}{b} \frac{b^{2}}{3 v^{2}} \cdot \Delta v \propto Y\left(\frac{m}{\rho}\right)^{3} \Delta v \ldots \ldots
$$

where $m$ is the mass of a fibrous mass compressed in a container and $\varrho$ the density of the fibers. Integrating this equation, he obtains the proportional relation between the pressure $p$ and the inverse cube of the height $v$.

In his experiments, Van Wyk observed the compression half of the final steady cycle after a number of compression and release cycles. Finding a discrepancy between theory and experiment in lower degrees of compression, he expressed an opinion which will be dealt with later in this article.

\section{Revision of Van Wyk's Theory}

In Van Wyk's theory the calculation of the mean value of $\mathrm{c}$ is the main subject of discussion. He determines the mean value of $c$ from the spatial integration based on the length of element, $b \cos \theta$, projected in the compressive direction-where $\theta$ is the angle between the direction of the fiber element and the compressive direction. We may say here that Van Wyk simply ignores the thickness of the fiber.

The following discussion is an attempt to make Van Wyk's theory more precise and to correct his estimate of $c$. The symbols used, except the new ones, are those cited in the synopsis. The total number of the elements in the container, all oriented at random, is $L / b$. Hence $\mathcal{N}_{\theta}$, the number of the elements oriented to $\theta$, is expressed by

$$
N_{\theta}=\frac{L}{b} \frac{2}{4 \pi} \sin \theta \cdot d \theta \int_{0}^{2 \pi} d \varphi,\left(0<\theta<\frac{\pi}{2}\right)
$$

In other words, $\mathcal{N}_{\theta}$ is proportional to the band area severed from the surface of a sphere of the unit radius by means of two cones having semi-vertical angles $\theta$ and $\theta+\Delta \theta$.

While other fibers contacting with those cited in the preceding paragraph are not necessarily oriented to the angle $\theta$, we may assume that only the elements identically oriented contact one another, if we reconstruct the real contacts. At this time the various entanglements, each consisting of only identically oriented elements, are superposed and spread over the container. When we pick out only an entanglement made up of elements of the orientation $\theta$, it is quite natural that the distribution of contact points in the entanglement of elements should cover uniformly the apparent volume of the specimen, and that the number of the contacts should still be statistically infinite.

The layer which has the vertical height of the elements oriented to $\theta$ may be thought of in the same light as in the synopsis. Therefore, the following relations can be formed, as in Van Wyk's theory, among $\Delta p_{\theta}, \Delta f_{\theta}$ and $\Delta y_{0}$ as regards the elements of the orientation $\theta$ :

$$
\begin{array}{r}
\Delta p_{\theta} \propto N_{\theta} \frac{c_{\theta}}{v} \Delta f_{\theta}, \Delta y_{\theta}=-\frac{c_{\theta}}{v} \Delta v, \\
\Delta f_{\theta} \propto \frac{I Y}{b^{3}} \Delta y_{\theta} \quad \cdots \cdots \cdots \cdots \cdots
\end{array}
$$

Connecting these, we neatly get the relation between $\Delta p$ and $\Delta v$ :

$$
\begin{aligned}
\Delta p=\Sigma \Delta p_{\theta} & =\Sigma\left(N_{\theta} \frac{c_{\theta}}{v}\right) \Delta f_{\theta} \\
& =-\frac{I Y}{b^{3}} \sum\left(N_{\theta} \frac{c_{\theta}^{2}}{v^{2}}\right) \Delta v
\end{aligned}
$$




$$
=-\frac{I Y}{b^{3}} \frac{L}{b} \frac{1}{v^{2}}\left[\int_{0}^{\pi / 2} c_{\theta}^{2} \sin \theta d \theta\right] \Delta v
$$

Unexpectedly, the integral term in this equation corresponds to the spatial mean of $c_{\theta}^{2}$. Use $c_{\theta}=$ $b \cos \theta$ in the above equation, and the result of the calculation will be the same as Van Wyk's. Howcver, our foregoing discussion makes it clear that: (a) The shares of pressure contributed by the elemental fibres need not be equal in value to elements oriented to any angle. Hence the need for equation (3), a somewhat unreasonable equation, lapses. (b) It is not $c$ but $c^{2}$ that requires the spatial mean. (c) It is inappropriate to express $c$ by $b \cos \theta$ which Van $W y k$ accepts.

Items (a) and (b) need no elaboration; they are self-evident from our discussion and equations. A few words about item (c). From equation (7) which contains $c_{\theta}$, it seems that Van Wyk loses sight of $\Delta p_{\theta}=\pi / 2$ and $\Delta y_{\theta}=\pi / 2$ contributed by the elements oriented nearly perpendicularly to the compressive direction. For, on $c_{\theta}=b \cos \theta, c_{\theta}$ equals zero with $\theta=\pi / 2$-which does not accord with the actualities.

It seems that this discrepancy originates from Van Wyk's lack of attention to the substantial thickness of a fiber when he expresses $c_{\theta}$. Fiber elements perpendicular to the compressive direction are small in number but they undoubtedly contribute more to compressive stress than fibers oriented otherwise do. To give a finite value to $\Delta p_{\theta}=\pi / 2$, it seems reasonable to give $c_{\theta}=\pi / 2$ fiber thickness $d$. Assuming that all the elements lie down perpendicularly to the container in a high degree of compression, the reduction in the thickness of the layer equals $(d \mid v) \cdot \Delta v$ and the number of the elements in the layer equals $\mathcal{N} d / v$, just as equation (7) shows. These facts attest to the reasonableness of our argument.

Imagine an elemental fiber with the substantial thickness taking a pose under a load. From Figure 1 the following equation is conceivable as the

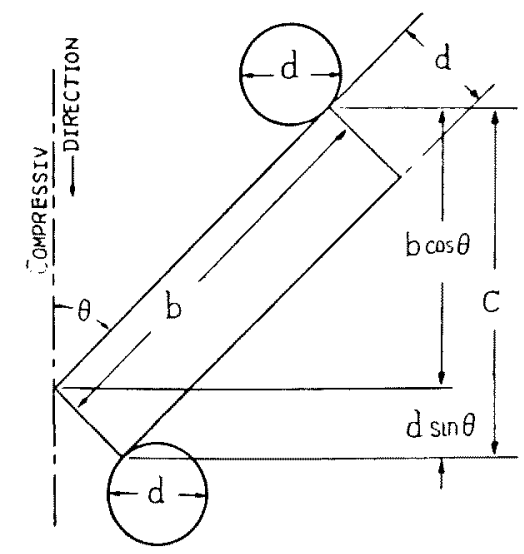

Fig. 1. Position of an elemental fiber orientated with $\theta$ in the compsresive direction. general expression of $c_{\theta}$, its square to be averaged later:

$$
c_{\theta}=b \cdot \cos \theta+d \cdot \sin \theta .
$$

This equation gives $c_{\theta}=d$ for $\theta=\pi / 2$, a fact which substantiates the authors' argument against Van Wyk's estimate. Substituting this equation into the equation (8), we get:

$$
\Delta p \propto-\frac{I Y}{b^{3}} \frac{L}{b} \frac{b^{2}+2 b d+2 d^{2}}{3 v^{2}} \cdot \Delta v
$$

Using equations (2) and (4) and integrating this equation (10) under the condition $v=$ const. at $p=0$, we derive:

$$
\begin{array}{r}
p \propto Y\left(\frac{2 m}{\rho}\right)^{3}\left\{\frac{1}{3} \frac{1}{v^{3}}+\frac{2}{4}\left(\frac{2 m}{\rho}\right) \frac{1}{v^{4}}\right. \\
\left.+\frac{3}{5}\left(\frac{2 m}{\rho}\right)^{2} \frac{1}{v^{5}}-\mathrm{const}\right\}
\end{array}
$$

This relationship between $p$ and $v$ differs by two terms having higher powers of $1 / v$ from Van Wyk's expression. The physical meaning of this equation is given by the following interpretation: Compare equation (10) with Van Wyk's, namely, equation (5) which disregards the thickness of the fiber; assume the formula

$$
\frac{\left(b^{2}+2 b d+2 d^{2}\right)^{\frac{1}{2}}}{v}=\frac{b}{v-l^{\prime}} ;
$$

and examine the significance of $l^{\prime}$. Superficially, this quantity $l^{\prime}$ supplements $v$ for the purpose of allowing $c_{\theta}$ to take $b \cdot \cos \theta$ which assumes fibers without thickness. Expanding equation (12) to a polynominal and marking the factor of this expansion, $v \cdot d / b$, with $l$, we get

$$
\begin{aligned}
l^{\prime}=l\{1 & -\frac{1}{2}\left(\frac{d}{b}\right) \\
& +\cdots \text { Polynomial terms of } d / b\} \cdots \cdots(
\end{aligned}
$$

The symbol $l$ denotes the substantial height of a fibrous mass in the container, because the relation, $l=v \cdot d \mid b=n \cdot d$, is formed from equation (2). The reason is that the value of $n \cdot d$ is the product of fiber thickness and the frequency with which the aforesaid sphere encounters fibers.

Since Van Wyk's estimate shows $d / b=1 / 6.5$ for merino wool, the sum of the higher-power terms in equation (13) is small compared with the first term. The symbol $l^{\prime}$ is, therefore, used to show the approximate value of $l$. In later sections $l$ is used in place of $l^{\prime}$

Substituting equation (12) into equation (10), we get:

$$
\begin{aligned}
\Delta p \propto-\frac{I Y}{b^{3}} & \frac{L}{b} \frac{b^{2}}{3\left(v-l^{\prime}\right)^{2}} \Delta v \propto-Y\left(\frac{2 m}{\rho}\right)^{3} \\
& \times \frac{1}{v^{2} \frac{\Delta v}{\left(v-l^{\prime}\right)^{2}}} \quad \cdots \ldots \ldots \ldots \ldots \ldots
\end{aligned}
$$


This result shows that Van Wyk's formula, i. e., equation (5) which makes no adjustment of $v$ in equation (14), overlooks the thickness of a fiber substance. Besides, only when his experiments in a high degree of compression disagreed with his theory did he see the need to adjust fiber thickness in his theoretical formula. It is obvious, however, that fiber thickness must be taken into account in any theory on the compressive process from the beginning to the end of that process. From this point of view our findings are more precise than Van Wyk's.

Van Wyk considers that the factors in his expression should be adjusted as a supplement to fiber thickness. In our theory we take the position that the factors must be corrected only for the possible deformation of the cross section of a fiber. Admittedly, as Van Wyk says, in actual cases diverse influences arise from factors left out of consideration, such as variations in staple length, diameter, contour, crimps and other elastic properties of a fiber.

\section{Experiments}

As expected, Van Wyk's method of experimenting consisted of putting a mass of wool fibers in a container, subjecting it to the usual experiment and observing the compression half of the final steady cycle. The hysteresis effect due to friction, he reckoned, was avoidable to a large extent. The results of his experiments apparently supported his theory on the proportionality of $p$ to $(1 / v)^{3}$ for moderate degrees of compression. Therefore, he extended his theory to variations in proportional factors attributable to the characteristics of a fiber.

We hold that the method of his experiment was unsuited to the conditions of his theory and that his theory over-extends itself. Our numerous laboratory experiments have shown that the wall of the container has a great deal to do with the results of the experiments. Essentially his theory, like ours, hold good of compression on specimens having an infinite lateral extent.

The method of compression test shown schematically in Figure 2 occurs to us as a more suitable method of experimenting to verify both theories. A

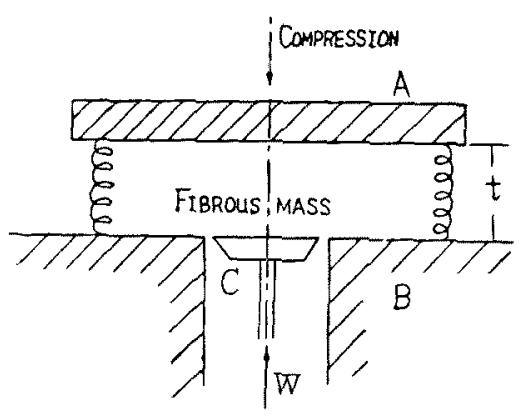

Fig. 2. Method of experiment. sample of a fibrous mass cylindrical in form and 15 $\mathrm{cm}$ in diameter is compressed between two plates, $A$ and $B$, and the distance $t$ observed as the height of the fibrous mass. Compressive force $W$ on a portion of the sample is measured with a circular plate $C$, which is partly separated from the bottom plate $B$ in its center. The surface of plate $C$ must always be kept on a level with the surface of plate $B$. Such a state can be brought about only by the use of an electric wire strain gauge (the displacement of $C$; $0.02 \mathrm{~mm}$ at maximum). This method of experiment probably accords with theorys because the fibers near the center line receive no lateral action.

Some examples of the results of experiments by this method for wool, wadding (cotton) and viscose rayon are given in Figures $3 a, 3 b, 3 b^{\prime}, 3 c$ and $3 c^{\prime}$, which show curves of pressure $W$ against several inverse powers of the height $t$ for the compression half of a cycle in a number of compression-release cycles. The sample mass was made of card web.

Figures $3 a, 3 b, 3 b^{\prime}, 3 c$ and $3 c^{\prime}$ generally show the possiblity that the pressure $W$ may be represented by a few terms of the polynomial of $1 / t$. Unlike Van Wyk's results, the concavities of curves for the terms of the same power are different for different samples. In the magnitude of the lateral bulging of the specimen, wool is the greatest, followed, in order, by viscose rayon and wadding cotton. From the results of these experiments we believe that our theory is preferable, but we have not determined the constants in our theoretical experssion from our experimental data. However, a comparison of the curves of the early and latter compressive cycles of viscose rayon and cotton is of special interest. The downward concavity of the curves increases progressively with the number of cycles. Perhaps this means, judging from equation (11), that the mass of the specimen just over the plate $C$ decreases due to bulging.

\section{Applicability of our Theory}

Considering the effect of friction on the so-called walled compression tests and the effect of building on the so-called wall-less compression tests, we can not but admit that our theory is applicable only to thin elemental layers of a specimen and that the effects of friction and bulging may be ignored or reckoned with in other ways. For this reason, Van Wyk's opinion merits attention so far as the lower degrees of compression are concerned.

Assuming that, no new contacts of fibers are formed and that therefore, $b$ remains constant, as Van Wyk thought it did in the Iower degrees of compression, the former equation in equation (14), derived by our revision, is transformed into

$$
p \propto Y\left(\frac{m}{v_{0}}\right)^{2}\left(\frac{m}{\rho^{3}}\right)\left(\frac{1}{v-l^{\prime}}-\frac{1}{v_{0}-l^{\prime}}\right)
$$

where $y=v_{0}$ at $p=0$. 
In this equation the shape of the pressure and volume curves is nearly hypabolic and the asymtote on the side of the lower degrees of compression is located in the region of $p<0$. These points agree fairly with the facts of the experiments. Van Wyk probably overlooked these points when he read his experimental curves.

(a) Wool

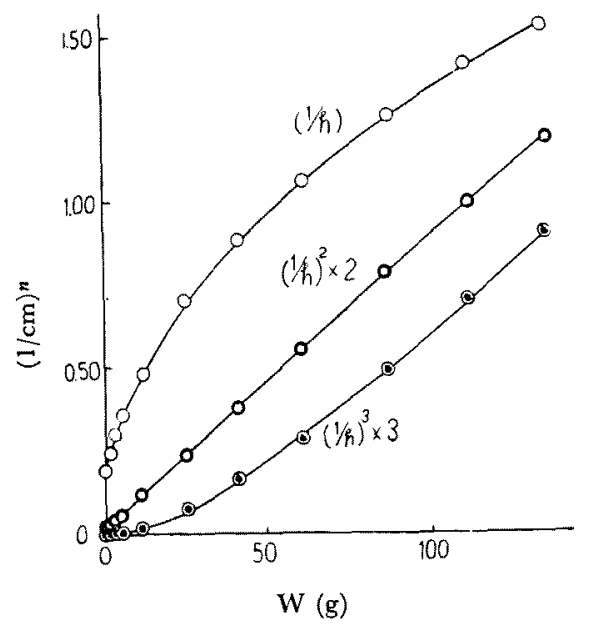

(b) Viscose rayon (the 6th cycle)

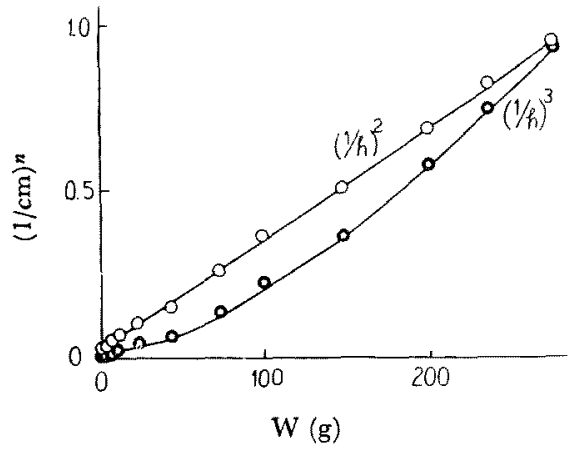

(b') Viscose rayon (the 16 th cycle)

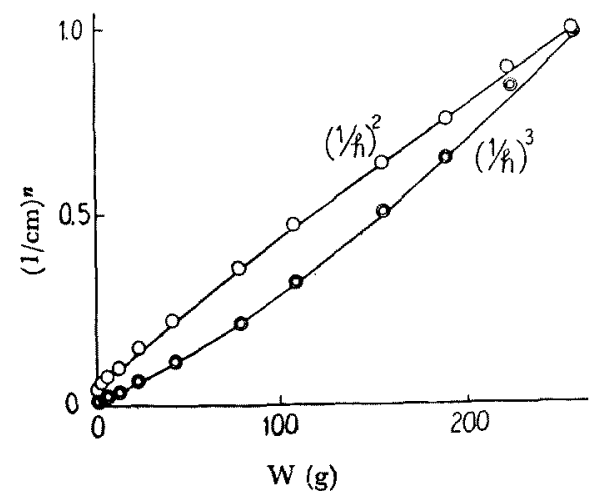

Equation (15) is conveniently applicable to an elemental thin layer in a sample mass. Notice the part of a fibrous mass just under the compressional piston and apply equation (15) to an elemental layer of any height in the specimen. Here the mass for the unit area may be rewritten in $\Delta m, v$ in the apparent thickness $\Delta t$, and $l^{\prime}$ in the substantial thickness $\Delta l$ (instead of $\Delta l^{\prime}$ ) as the respective quantities with regard to the respective layers. The relation $\Delta m=\gamma \cdot \Delta l$ is then reasonably established with a proportional factor $\gamma$. Equation (15), therefore, is rewritten thus:

$$
p=k Y\left(\frac{\Delta m}{\Delta t_{0}}\right)^{2} \frac{\gamma}{\rho^{3}}\left(\frac{\Delta l}{\Delta t-\Delta l}-\frac{\Delta l}{\Delta t_{0}-\Delta l}\right),
$$

where $k$ is an unknown proportional factor.

(c) Cotton for wadding (the 2nd cycle)

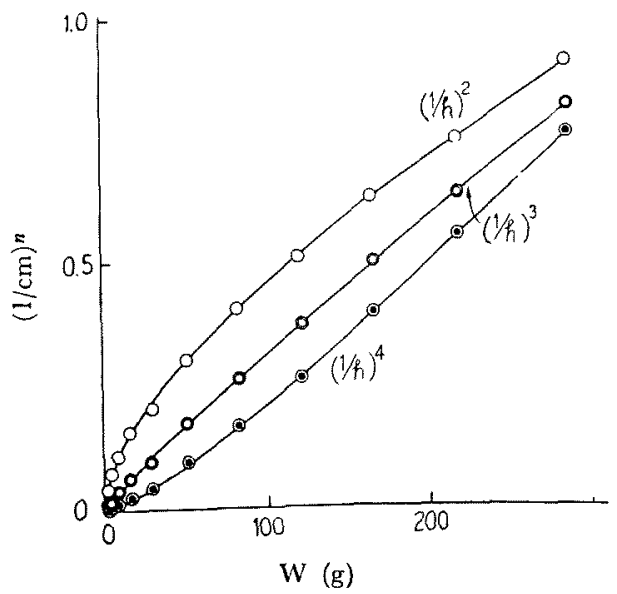

(c') Cotton for wadding (the 17th cycle)

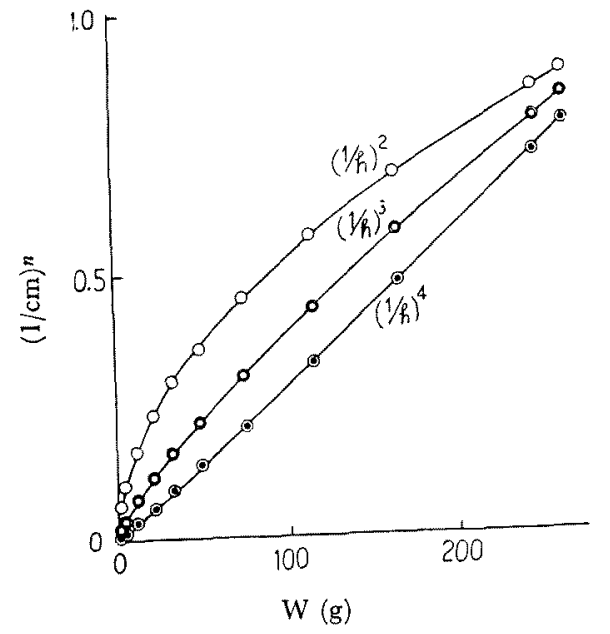

Fig. 3. Relations between the compressive force and the several inverse powers of apparent heights of various specimens under compression. 
The equation can be neatly transformed into this useful from:

$$
\frac{\Delta l}{\Delta t-\Delta l}=\frac{1}{\lambda}\left(\omega_{0}+\frac{\text { compressive force }}{\text { area of piston }}\right) \cdots
$$

where

$$
\begin{aligned}
& \lambda=k Y\left(\frac{\Delta m}{\Delta t_{0}}\right)^{2} \frac{\gamma}{\rho^{3}} \\
& \left.\omega_{0}=\lambda \frac{\Delta l}{\Delta t_{0}-\Delta l}\right)
\end{aligned}
$$

$\Delta t-\Delta l$ being distinctly a clearance in the layer, the left side of equation (17) denotes the degrees of the packing of the fibers. The symbols $\lambda$ and $\omega_{0}$ mean the constant values of the layer consisting of (1) initial values $\Delta m / \Delta t_{0}$ of the density of packing, (2) the initial values of the degree of packing, (3) Y, (4) $\gamma$, and (5) $\varrho$ - the latter three being, respectively, the characteristic values of the fibers in the layer.

Equation (17) gives us the valuable information that the degree of packing relative to an elemental layer of a fibrous mass varies proportionally to the pressure in which the so-called latent pressure $\omega_{0}$ is taken into account. The proportional constant $\lambda$ can be named "the modulus of the degree of packing." Of course, the relation can be effective only in lower degrees of compression, and has presumably been used for practical purposes without theoretical backing.

Where a specimen is uniform before compression and equations (2) and (4) are used, equation
(18) takes this simpler form:

$$
\left.\begin{array}{l}
\lambda=\frac{k Y}{2}\left(\frac{m}{t_{0}}\right)^{2} \frac{1}{\rho^{2}} \\
\omega_{0}=\lambda \frac{l}{t_{0}-l}
\end{array}\right\}
$$

and these values show the characteristic constant values of the specimen under compression. Equation (17), therefore, becomes the integral-possible form along the height of the specimen. At this time the lateral conditions of the specimen must be transformed into a pressure and added to the pressure term in equation (17). The applicability of this concept will be dealt with in a future issue.

\section{Summing up}

Van Wyk's theory as regards the pressure-height relation of a compressed fibrous mass has been partly corrected and developed, with the corrections and developments shown by experiments. Our countertheory as it now stands may not be directly applicable to the usual compression test, but it has, meanwhile, developed useful information. That information is applicable only to the low stage of compression.

\section{Literature cited}

[1] C. M. van Wyk; 7. Text. Inst., Vol. 37, No. 12, T. 285 (1946) [2] P. G. de Macarty; Text. Res. J., Vol. 25, No.10, p. 875 (1955) 